\title{
Combined Ortho-Surgical Treatment of A Patient With Facial Asymmetry
}

\author{
Siddharth Mehta, Abhay T Kamath, G Srikanth and Adarsh kudva \\ Department of Orthodontics and Dentofacial Orthopedics, Mahatma Gandhi dental college, India
}

Received: 琒 May 21, 2018; Published: 眥 May 31, 2018

*Corresponding author: Siddharth Mehta, Department of Orthodontics and Dentofacial Orthopedics, Mahatma Gandhi dental college, MGUMST, Jaipur, India

\begin{abstract}
3D treatment planning helps to assess the position of a jaw in all the dimensions and plan surgery. Rapid prototyping technology allows for rapid construction of bony models. The present case report shows the use of stereolithographic model to plan bilateral sagittal split osteotomy for patient with facial asymmetry.
\end{abstract}

Keywords: Facial asymmetry; Rapid prototyping; Stereolithographic model

\section{Introduction}

The aim of orthognathic surgery is to treat any jaw imbalances, bring aboutan improvementin bite, which could otherwise adversely affect the esthetics and function. 3 dimensional movements can be performed by model surgery which helps in the fabrication of inter-occlusal splint needed for achieving good occlusion and function. However, the outcome of model surgery depends upon the expertise of the individual as well as accuracy of movements $[1,2]$. The recent advances in digital technology help to overcome such limitations. Rapid prototyping is one such technique that helps fabricate stereolithographic models from images captured by cone beam computed tomography or magnetic resonance imaging [3]. This method has 2 stages: one virtual which involves preparing a simulation model through various image processing tools and second is generation of a physical model through computer aided designing (CAD) [4].

\section{Case Report}

A patient reported to the Department of Orthodontics with a clicking sound on right TMJ, difficulty in opening mouth as well as deviation of the lower jaw. He reported no history of trauma but had undergone orthodontic treatment 2 years back. He had undergone lower first premolar extraction but discontinued orthodontic treatment in between. His extra-oral examination revealed a straight profile and chin point deviated to the right side by $3 \mathrm{~mm}$ in the frontal view. Smile analysis showed maxillary arch canted to the left side. Intraoral examination showed class III incisor relation, lower first premolars extracted, a class III molar on the left side, a class I molar relation on the right side and entire cross bite with respect to right side of the jaw. Lower midline was deviated to the right side by $3.5 \mathrm{~mm}$.

Final diagnosis for the patient was skeletal class III and dental Angle's class III subdivision left side malocclusion with chin point deviated to right side by $3 \mathrm{~mm}$ and cross bite with respect to right side and lower midline deviation by $3.5 \mathrm{~mm}$ to right side. Cephalometric records (Figure 1) and plaster models were prepared.

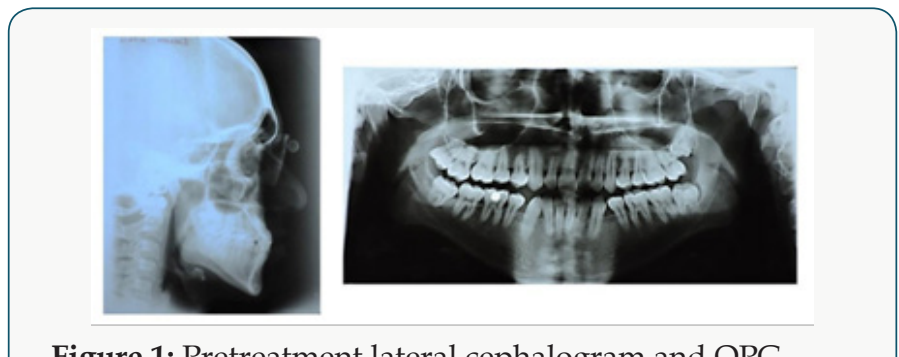

Figure 1: Pretreatment lateral cephalogram and OPG.

\section{Treatment objectives}

a) Coordination of upper and lower arches

b) Correction of class III skeletal pattern and asymmetry

c) Correction of Maxillary cant

d) Correction of cross bite and improvement in bite 
e) Achievement of class I incisor relationship

\section{Treatment plan}

a) Combined surgical orthodontic plan to correct the bite and malocclusion

b) Muscle deprogramming

c) Alignment and coordination of upper and lower arches

d) Surgery - Asymmetric set back and rotation of the mandibular arch Post-surgical finishing and debonding

\section{Treatment progress}

Pre-surgical procedure: Treatment started with muscle deprogramming with a butterfly deprogrammer (Figure 2). After 6 weeks of the appliance wear, the patient reported an improvement during opening and closing of jaw. The mouth opening also improved and clicking sound disappeared. This was followed by the placement of $0.022^{\prime \prime}$ preadjusted edgewise brackets and molar tubes (MBT Victory series, 3M,

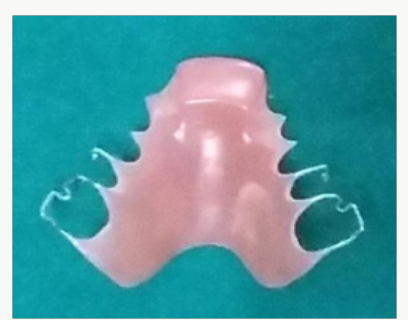

Figure 2: Butterfly deprogrammer.

UNITEK, Monrovia, CA, USA). Leveling and alignment with $0.016^{\prime \prime}$ NiTi archwire (d-tech, Wagholi, Pune, India) and wellcoordinated 0.018" stainless steel (S.S.) arch wires (A.J. Wilcock Premium Plus Australian arch wire, Whittlesea, Victoria, Australia) was done. During the alignment phase almost 6 months before the planned surgery, lower third molars were extracted. 0.019x0.025" stainless steel wire (Truforce, Ortho Technology, Inc., Tampa, USA) was then placed with hooks to finish the pre-surgical phase (Figure 3). Patient was then referred to Department of Oral surgery.
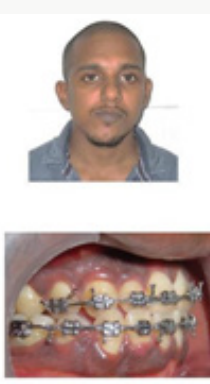
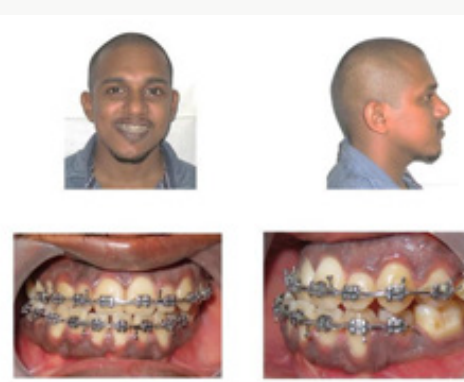

Figure 3: Pre-surgical intra oral and extra oral photographs.

On the day surgery, patient reported with debonded molar tubes due to a minor accident. Further plan of taking a pre-surgical CT scan was then performed (Figure 4) and a stereolithographic model was prepared from the same. After planning mock surgery on patient's casts and rapid prototype model, an acceptable bite was established by performing a rotational BSSO (bilateral sagittal split osteotomy). Patient insisted on a single jaw surgery, so the plan was designed to correct the mandibular deviation and maxillary cant was left untouched (Figure 5).
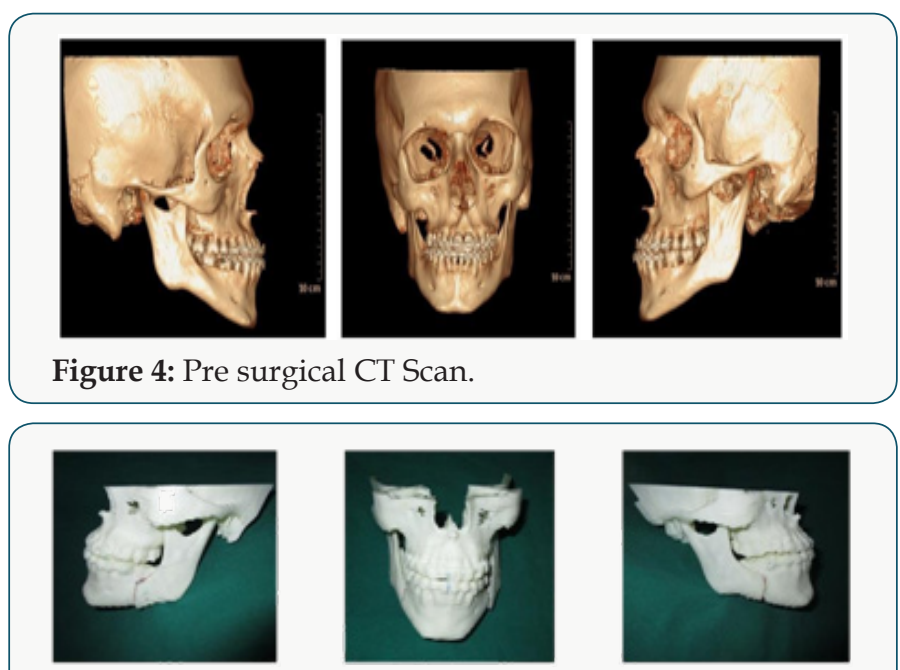

Figure 5: Stereolithographic model prepared from CT scan and mock surgery performed.

\section{Surgical procedure}

Under aseptic precaution after securing adequate general anesthesia, local anesthesia was given bilaterally in the posterior mandibular region. Soft tissue incision was given first on right side along the anterior border of the ramus of mandible up to first molar region. Incision was done subperiosteally to expose the coronoid notch superiorly. Markings were made for osteotomy cut with pencil and steps were followed in the same sequence as model surgery on stereolithographic model. First horizontal corticotomy cut was given along the medial aspect of the mandible above the mandibular canal. Second vertical corticotomy cut was given between internal and external oblique ridge continuing along the lateral aspect of mandible up to distal aspect of first molar. Third vertical osteotomy cut was given along the body of mandible from lower border of the mandible joining the intermediate cut superiorly. Then, the center corticotomy cut was given and osteotomy was completed. Similar steps were performed on the left side. The proximal and distal segments were mobilized and nerve was identified in the distal segment. The teeth were guided into desired occlusion and maxillo-mandibular fixation was done using 26 gauge wire. Thus, a rotational BSSO was performed. Condyle was guided into its centric position using condylar guiding device holding the distal part of proximal segment superiorly and posteriorly. 4 Bur holes ( 2 on either side of osteotomy) of $1.6 \mathrm{~mm}$ in diameter were drilled. Mandibular plate fixation was done with screws (2x8mm); after which the inter-maxillary fixation (IMF) was released and occlusion was reassessed on opening and closing the mouth which was found to be satisfactory. Patient recovered well from the anesthesia and was shifted to post-operative ICU. 


\section{Post-surgical procedure}

Post surgically, a class 1 canine on right side and class 3 molar relation bilaterally was achieved, however, end on canine was achieved on the left side. Slight maxillary canting was present, however patient refused for further intervention. The patient was asked to maintain the appliance for next 6 months to check for the stability of treatment. After 6 months the appliance was debonded and a fixed lingual retainer was bonded in the lower arch and a removable Hawley's retainer in the upper was given (Figures 6 \& 7). The patient was advised to get the upper third molars extracted.
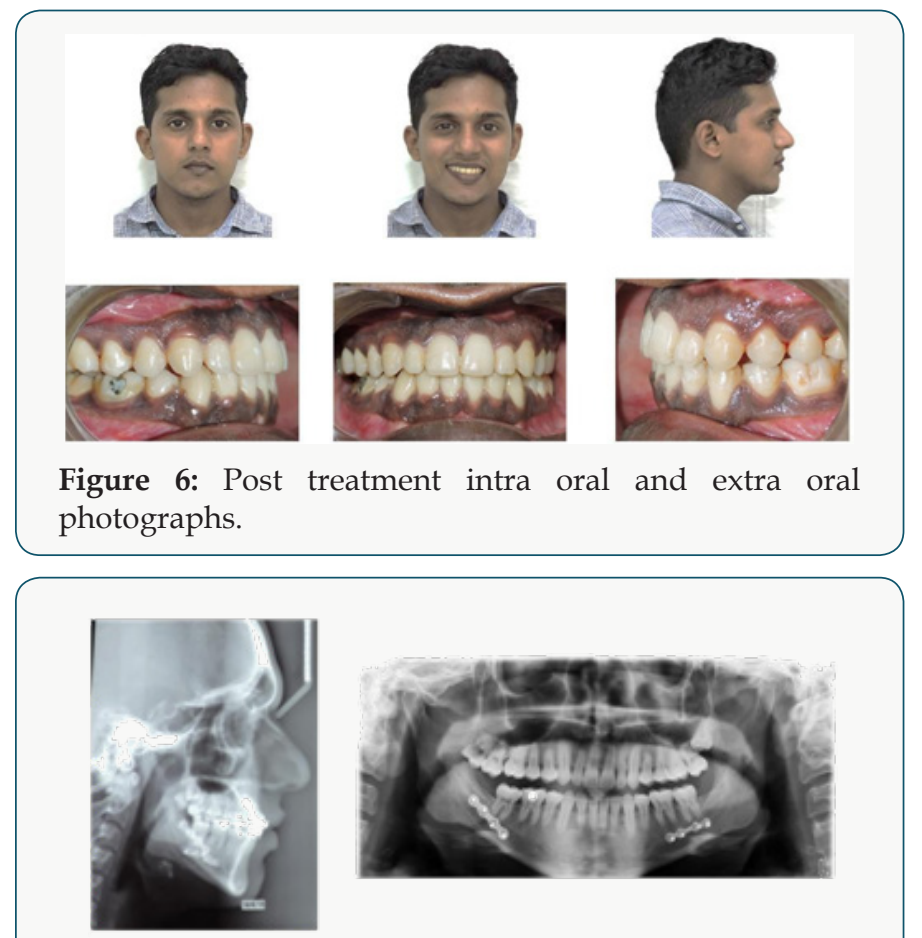

Figure 7: Post treatment lateral cephalogram and OPG.

\section{Results}

The first molars were successfully brought into a Class-III relationship with correction of cross bite. Treatment time was 2 years. A comparison of postero-anterior cephalogram revealed an improvement in chin point deviation (Figure 8). End of treatment, a proper overjet and overbite with improvement in appearance as well function was achieved. Patient reported lack of clicking sound and an improvement in bite.
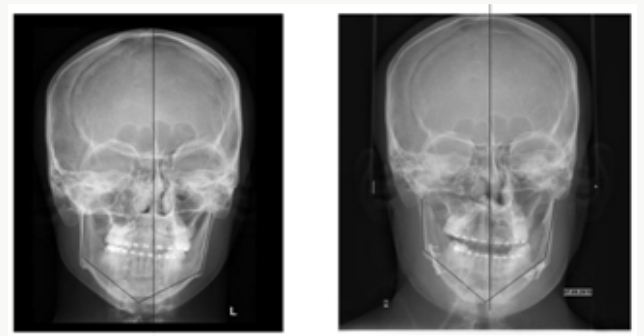

Figure 8: Comparison of PA cephalogram pre and postsurgery.

\section{Discussion}

Development of high-resolution computed imaging and modelling technologies has been extremely useful when diagnosing and planning the treatment of maxillofacial deformities. Mankovich et al [5] was the first to report the use of rapid prototyping technology for construction of bony models. Stereolithographic models are three-dimensional (3D) models that allow 3 dimensional assessments of hard tissue structures. These models are made of polypropylene/ABS-like material. Other materials available are epoxy and acrylic resins. Selective coloring of the vital structures is also possible by a technique of color stereolithography, which allows to mark out important vital structures. This method has been widely applied as an aid in patients with complex heart diseases [6], cochlear implant surgery [7], cranio-maxillofacial surgery [8], trauma cases [9], placing dental implants [10] and even designing attachments for impacted canine [11].

In maxillofacial surgery, the accurate replicas help in carrying out mock surgery, determining site of saw placement, bur drilling and screw fixation, which can then be carried out on the table. In this case, mandibular surgery was planned on the stereolithographic model. Mock surgery was performed and the plates were contoured on the stereolithographic models. The mandible was rotated into the correct position following bilateral sagittal split osteotomy. The facial asymmetry improved significantly and stable occlusion was achieved.

\section{Conclusion}

Rapid prototyping allows clinicians to plan orthognathic surgeries precisely in patients with facial asymmetry and helps save a lot of operation theatre time.

\section{References}

1. Bill JS, Reuther JF, Dittmann W, Kübler N, Meier JL et al. (1995) Stereolithography in oral maxillofacial operation planning. Int J Oral Maxillofac Surg 24(1 Pt 2): 98-103.

2. Hibi H, Sawaki Y, Ueda, M (1997) Three-dimensional model simulation in orthognathic surgery. Int J Adult Orthod Orthognathic Surg 12(3): 226-232.

3. James WJ, Slabbekoom MA, Edgin WA, Hardin C (1998) Correction of congenital malar hypoplasia using stereolithography for presurgical planning. J Oral Maxillofac Surg 56(4): 512-517.

4. Everton Luis Santos da Rosa, César Fernando Oleskocicz, Bruno Nogueira Argao (2004) Rapid Prototyping in Maxillofacial Surgery and Traumatology: Case Report. Braz Dent J 15(3): 243-247.

5. Mankovich NJ, Cheeseman AM, Stoker NG (1990) The display of three dimensional anatomy with stereolithographic models. J Digital Imaging 3(3): 200-203.

6. Stephan Jacobsa, Ronny Grunertb, Friedrich W Mohra, Volkmar Falka (2008) 3D-Imaging of cardiac structures using 3D heart models for planning in heart surgery: a preliminary study. Interact CardioVasc Thorac Surg 7(1): 6-9.

7. Lopponen H, Holma T, Sorri M, Jyrkinen L, Karhula V, et al. (1997) Computed tomography data based rapid prototyping model of the 
temporal bone before cochlear implant surgery. Acta Otolaryngol Suppl 529: 47-49.

8. Xia D, Gui L, Zhang Z, Lu C, Niu F, et al. (2005) Fabrication of 3-dimensiona skull model with rapid prototyping technique and its primary application in repairing one case of cranio-maxillo-facial trauma. Zhongguo Xiu Fu Chong Jian Wai Ke Za Zhi 19(10): 811-814.

9. Christian Herlin, Matthieu Koppe, Jean-Luc Béziat, Arnaud Gleizala (2011) Rapid prototyping in craniofacial surgery: Using a positioning guide after zygomatic osteotomy-A case report Journal of CranioMaxillofacial Surgery 39(5): 376-379.

10. Kianoosh Torabi, Ehsan Farjood, Shahram Hamedani (2015) Rapid Prototyping Technologies and their Applications in Prosthodontics, a Review of Literature. J Dent (Shiraz) 16(1): 1-9.

11. Jorge Faber, Patrícia Medeiros Berto, Marcelo Quaresma (2006) Rapid prototyping as a tool for diagnosis and treatment planning for maxillary canine impaction. Am J Orthod Dentofacial Orthop 129: 583-589.

\section{(c) (i) Commons Attribution 4.0 License}

To Submit Your Article Click Here:

Submit Article

DOI: 10.32474/MADOHC.2018.02.000139

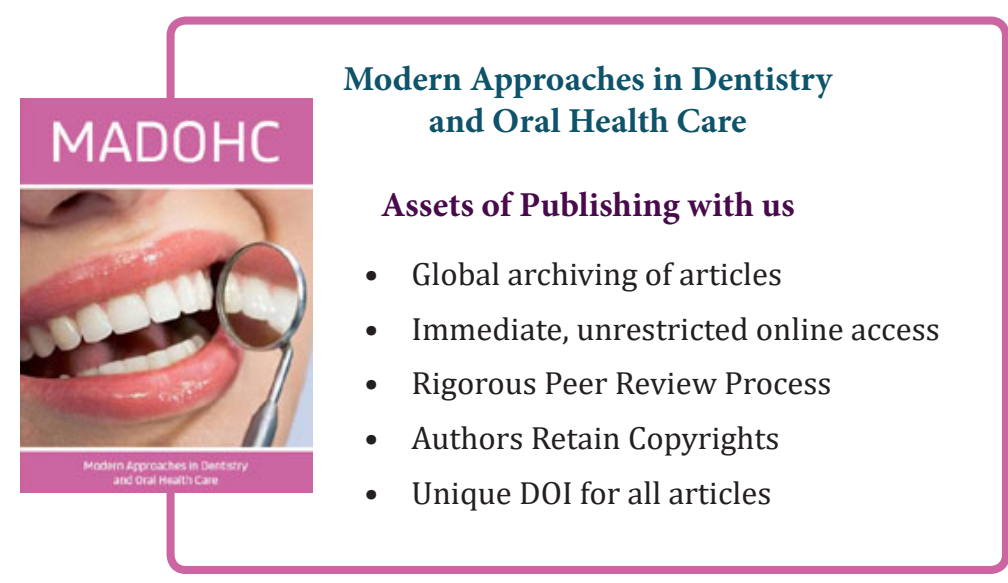

